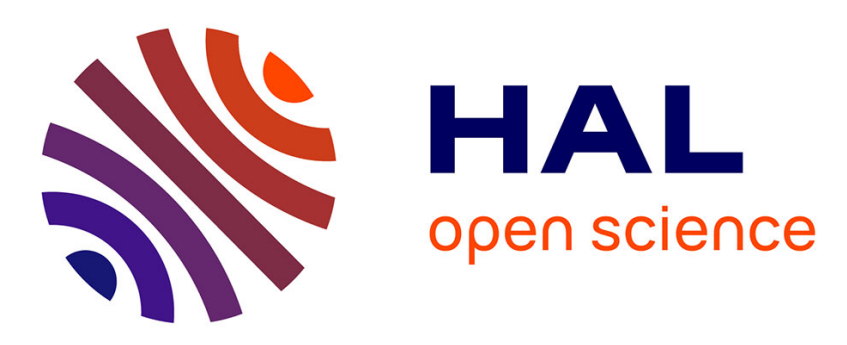

\title{
Nonuniform switching of the perpendicular magnetization in a spin-torque-driven magnetic nanopillar
}

D. P. Bernstein, Björn Bräuer, Roopali Kukreja, Joachim Stöhr, Thomas

Hauet, Julien Cucchiara, Stéphane Mangin, J. A. Katine, Tolek Tyliszczak, K.W. Chou, et al.

\section{To cite this version:}

D. P. Bernstein, Björn Bräuer, Roopali Kukreja, Joachim Stöhr, Thomas Hauet, et al.. Nonuniform switching of the perpendicular magnetization in a spin-torque-driven magnetic nanopillar. Physical Review B: Condensed Matter and Materials Physics (1998-2015), 2011, 10.1103/PhysRevB.83.180410 . hal-01345380

\section{HAL Id: hal-01345380 \\ https://hal.science/hal-01345380}

Submitted on 13 Jul 2016

HAL is a multi-disciplinary open access archive for the deposit and dissemination of scientific research documents, whether they are published or not. The documents may come from teaching and research institutions in France or abroad, or from public or private research centers.
L'archive ouverte pluridisciplinaire HAL, est destinée au dépôt et à la diffusion de documents scientifiques de niveau recherche, publiés ou non, émanant des établissements d'enseignement et de recherche français ou étrangers, des laboratoires publics ou privés. 


\title{
gु \\ Nonuniform switching of the perpendicular magnetization in a spin-torque-driven magnetic nanopillar
}

\author{
David P. Bernstein, Björn Bräuer, Roopali Kukreja, and Joachim Stöhr \\ Stanford Institute for Materials and Energy Science, SLAC National Accelerator Laboratory, Menlo Park, California 94025, USA
}

Thomas Hauet

Institut Jean Lamour, CNRS-Nancy Université-UPV Metz, Vandoeuvre lés Nancy, France and Hitachi Global Storage Technologies, San Jose, California 95119-1103, USA

Julien Cucchiara and Stéphane Mangin

Institut Jean Lamour, CNRS-Nancy Université-UPV Metz, Vandoeuvre lés Nancy, France

Jordan A. Katine

Hitachi Global Storage Technologies, San Jose, California 95119-1103, USA

Tolek Tyliszczak and Kang W. Chou

Advanced Light Source, Lawrence Berkeley National Laboratory, California 94720, USA

Yves Acremann

Laboratory for Solid State Physics, ETH Zurich, Switzerland

(Received 6 October 2010; revised manuscript received 20 April 2011; published 10 May 2011)

\begin{abstract}
Time-resolved scanning transmission x-ray microscopy measurements were performed to study the currentinduced magnetization switching mechanism in nanopillars exhibiting strong perpendicular magnetic anisotropy. This technique provides both short-time $(70 \mathrm{ps})$ and high-spatial $(25 \mathrm{~nm})$ resolutions. Direct imaging of the magnetization demonstrates that, after an incubation time of $\sim 1.3 \mathrm{~ns}$, a $100 \times 300 \mathrm{~nm}^{2}$ ellipsoidal device switches in $\sim 1 \mathrm{~ns}$ via a central domain nucleation and opposite propagation of two domain walls toward the edges. High domain-wall velocities on the order of $100 \mathrm{~m} / \mathrm{s}$ are measured. Micromagnetic simulations are shown to be in good agreement with experimental results and provide insight into magnetization dynamics during the incubation and reversal periods.

DOI: 10.1103/PhysRevB.83.180410

PACS number(s): 75.78.Jp, 75.30.Gw, 75.70.Kw, 78.20.Ls
\end{abstract}

Spin-polarized current-induced magnetization switching (CIMS) has now been reported in many experimental works involving a wide variety of geometries including point contacts, nanopillars (spin valves or tunnel junctions), and nanowires with or without notches. ${ }^{1}$ These systems are extensively studied, in part, because they hold the potential for applications in spin-transfer magnetic random access memory. ${ }^{2}$ Interest in materials with perpendicular magnetic anisotropy (PMA) has grown considerably as a pathway for lowering the critical current required to switch the magnetization while maintaining thermal stability as compared with in-plane systems. ${ }^{3,4}$ This interest has, to a large degree, stemmed from calculations of the switching behavior using a macrospin approximation. ${ }^{5}$ Although reproducible ultrafast switching with high efficiency has been demonstrated, ${ }^{6}$ the macrospin model has not been consistent with all experimental results.

To fully understand CIMS dynamic phenomena in PMA materials, two time scales need to be considered: a long-time scale dominated by thermal activation ${ }^{7,8}$ and a short-time scale dominated by angular momentum conservation. ${ }^{6,7}$ Early studies on spin transfer in PMA-based devices have been devoted to a description of quasistatic phenomena on longer time scales. Only very recent experiments have used time-resolved transport measurement with current pulses as short as 300 ps. $^{6,7}$

In this Rapid Communication, we present the results obtained by studying the CIMS mechanism in PMA nanopillars at nanometer length scales and picosecond time scales using scanning transmission x-ray microscopy (STXM) images taken while injecting a spin-polarized current. These images are further compared with micromagnetic simulations. We demonstrate that, in $100 \times 300 \mathrm{~nm}^{2}$ devices, CIMS starts with preswitching dynamics that result in the nucleation of a reversed magnetic domain. The switching mechanism is completed by two domain walls propagating in opposite directions and their subsequent annihilation at the edge of the pillar. The delay time, domain nucleation, and domainwall propagation are discussed. A domain-wall velocity of $\sim 100 \mathrm{~m} / \mathrm{s}$ is measured and is shown to be consistent with micromagnetic simulations. ${ }^{9}$

The multilayer samples were deposited by dc magnetron sputtering at 2 mTorr Ar pressure onto ambient-temperature $\mathrm{Si}$ wafers coated with $\mathrm{Si}_{3} \mathrm{~N}_{4}$. The reference and free magnetic layers of the sample stack are $\mathrm{Pd}(2 \mathrm{~nm}) /[\mathrm{Co}(0.29 \mathrm{~nm}) / \mathrm{Pd}$ $(0.8 \mathrm{~nm})] \times 5 /[\mathrm{Co}(0.2 \mathrm{~nm}) / \mathrm{Ni}(0.7 \mathrm{~nm})] \times 2 / \mathrm{Co}(0.2 \mathrm{~nm})$ and $[\mathrm{Co}(0.2 \mathrm{~nm}) / \mathrm{Ni}(0.7 \mathrm{~nm})] \times 5 / \mathrm{Co}(0.2 \mathrm{~nm})$, respectively. A $4 \mathrm{~nm} \mathrm{Cu}$ spacer layer was deposited to ensure a magnetic decoupling between the two magnetic layers. Top and bottom leads are $\mathrm{Ta} / \mathrm{Cu}(50 \mathrm{~nm})$ and $\mathrm{Cu}(15 \mathrm{~nm}) / \mathrm{Ta}$ $(3 \mathrm{~nm})$, respectively. The multilayer film was then patterned using electron-beam lithography and ion etching to form $100 \times 300 \mathrm{~nm}^{2}$ nanopillars with an ellipsoidal shape. A last chemical etching step was performed on the sample backside to 


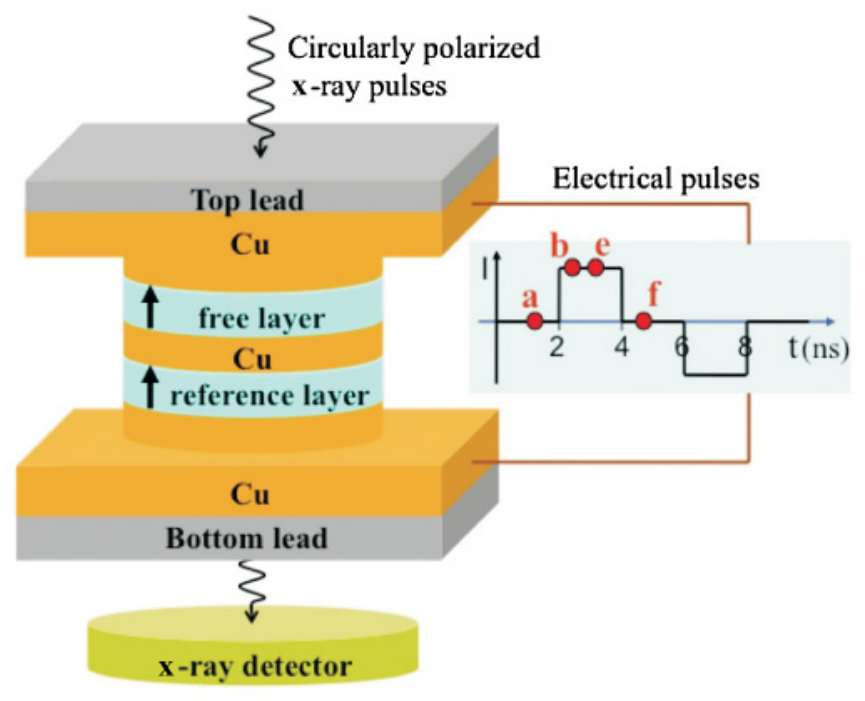

FIG. 1. (Color online) Schematic of the STXM experiment performed on a $[\mathrm{Co} / \mathrm{Ni}]$-based nanopillar spin valve. The pulse sequence (current versus time) is shown on the right. Letters (a), (b), (d), and (f), respectively, represent the STXM measurements corresponding to the images shown in Fig. 2.

open a $200 \times 200 \mathrm{~nm}^{2}$ window in the $\mathrm{Si}$ wafer. The spin-torque devices sit in the center of this window on a 300 nm-thick $\mathrm{Si}_{3} \mathrm{~N}_{4}$ membrane chosen for its transparency to X-rays. The samples were finally mounted and wire bonded to custom printed circuit boards so that current pulses could be injected.

In order to image the magnetization reversal process, the STXM available on beamline 11.0.2 at the Advanced Light Source (ALS) was used. Images of the spatially resolved magnetic contrast with a time resolution of 70 ps were obtained as described in Refs. 10-13. Beamline 11.0.2 is equipped with an elliptically polarized undulator capable of providing arbitrary circular or linear polarization. In our experiment, the incident beam was parallel to the surface normal as shown in Fig. 1 and was focused by a zone plate with a $25 \mathrm{~nm}$ resolution. The photon energy is tuned to the characteristic $\mathrm{Ni} \mathrm{L}_{3}$ resonance edge. Note that $\mathrm{Ni}$ is present in both free and reference layers but is 2.5 times more in quantity in the free layer. In the following, we consider the magnetic configuration of the high-anisotropy reference layer uniform and fixed, as confirmed by the simulation. The experiment is repeated for both left- and right-circular polarizations to provide a magnetic contrast through the $\mathrm{x}$-ray magnetic circular dichroism effect. ${ }^{14}$ The experiment provides, for one specific element (nickel in our case), a measurement of the magnetization component parallel to the incident light direction, i.e., along the PMA axis.

A critical feature of our experimental setup is the ability to synchronize the current pulses to the bunch structure of the ALS ring within a precision of $70 \mathrm{ps}$, yielding the time resolution of our experiment. ${ }^{10,11}$ As presented in Fig. 1, the current pulse sequence was applied as follows: 4 ns positive or set pulse/4 ns no current/4 ns negative or reset pulse/4 ns no current. Voltages of $\pm 748 \mathrm{mV}$ were applied during the set and reset pulses, respectively, corresponding to a current density of $\sim 5 \times 10^{7} \mathrm{~A} / \mathrm{cm}^{2}$. The rise time of the current pulses was $100 \mathrm{ps}$. By varying the delay between the $\mathrm{x}$-ray probe and the current pulses, we were able to measure the time evolution of the magnetization. Figure 2 shows a typical switching event as electrons traveled from the free layer to the reference layer. Therefore, the imaged CIMS process corresponds to the free-layer magnetization switching from a parallel to an AP alignment with respect to the reference layer magnetization. The experiment was carried out at room temperature and in the absence of a magnetic field.

The preset image in Fig. 2(a) shows the free-layer magnetization state before a current pulse is injected into the structure. Images shown in Fig. 2 are obtained by accumulating data while repeating the method described above. It is important to note that, since our experiment uses STXM in a pump-probe mode, the images show the perpendicular component of the $\mathrm{Ni}$ sublattice magnetization averaged over a large number of pulse sequences. As a consequence of the averaging, this method does not allow us to identify stochastic processes. Figure 2(b) shows that the sample undergoes a so-called incubation time during which the pillar is subjected to a spin-polarized current, but the free layer has not started to reverse its magnetic orientation. ${ }^{15,16}$ After this incubation time, a central region of the pillar starts reversing. This region of nucleation appears more clearly after 50 ps in Fig. 2(c). Times quoted in Fig. 2 are given with respect to the first observed domain nucleation [Fig. 2(b)] because of a possible offset in the absolute time.

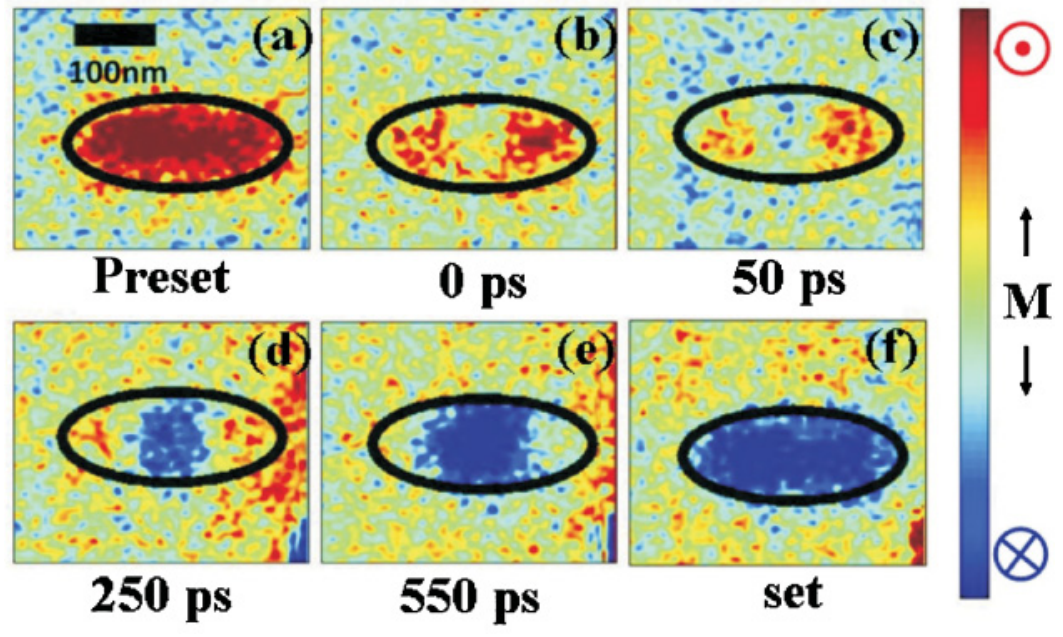

FIG. 2. (Color) Experimental STXM images of the magnetic contrast in a $100 \times 300 \mathrm{~nm}^{2}$ ellipsoidal nanopillar spin valve. Images (a)-(e) have been taken at different times during the CIMS reversal based on the setup shown in Fig. 1. Image (a) is the initial state, and (f) is the final state. The color scale corresponds to the perpendicular component of the free-layer magnetization, from parallel $(\mathrm{P})(\mathrm{red})$ to antiparallel (AP)(blue) with respect to the reference layer. 
In other words, the precision on the pulse onset time does not allow us to quantify the $1.3 \mathrm{~ns}$ incubation time with a better precision than $300 \mathrm{ps}$. The relative times, however, are accurate within the quoted time resolution. In Figs. 2(d) and 2(e), the reversed domain grows by domain-wall motion toward the edges. In Fig. 2(e), at 550 ps, the sample magnetization has not fully switched yet. An image taken in between the set pulse and the reset pulse confirms the total saturation of the sample [Fig. 2(f)]. The present data set offers direct proof of incoherency in the short-time regime CIMS. It provides an explanation for the discrepancies between the macrospin model and the very recent time-resolved macroscopic transport measurements performed on similar PMA devices. ${ }^{6-8}$

To get a better understanding of the magnetization reversal, we performed a three-dimensional micromagnetic calculation of the Landau-Lifshitz-Gilbert (LLG) equation using the Scheinfein code $^{17}$ where the injected current pulse was taken into account in the Slonczewski spin-torque term. ${ }^{18}$ The calculations performed considered a $100 \times 300 \mathrm{~nm}^{2}$ ellipsoidal element mimicking the same stack as described above where the reference layer is divided into 3750 cells and the soft layer is divided into 1875 cells. The free-layer parameters were $M_{s}=650 \mathrm{emu} / \mathrm{cm}^{3}(0.650 \mathrm{~A} / \mathrm{m})$ and $K_{u}=$ $2.7 \times 10^{6} \mathrm{erg} / \mathrm{cm}^{3}\left(2.7 \times 10^{5} \mathrm{~J} / \mathrm{m}^{3}\right)$. The reference layer had a saturation magnetization $M_{s}=500 \mathrm{emu} / \mathrm{cm}^{3}$, and its magnetization was kept fixed along the anisotropy axis perpendicular to the film plane. The intralayer exchange coupling between cells is $2 \mu \mathrm{erg}(20 \mathrm{pJ} / \mathrm{m})$ (for both layers), the current polarization is $p=0.35$, and the damping coefficient is $\alpha=0.1{ }^{3}$ Temperature was taken into account within an initial $5^{\circ}$ tilt of the free-layer magnetization. Current duration and amplitude were $4 \mathrm{~ns}$ and $15 \mathrm{~mA}$, respectively. The electrons were injected from the free to the hard layer starting with a parallel initial configuration. A sequence of those simulations is shown in Fig. 3.

The micromagnetic simulations shown in Figs. 3(a)-3(g) and the experimental STXM results are in good qualitative agreement. The simulated incubation time and switching time are 1.3 and $1.2 \mathrm{~ns}$, respectively. The size of the nucleated domain is smaller than $50 \times 50 \mathrm{~nm}^{2}$, and the domain-wall width is on the order of $20 \mathrm{~nm}$. The simulated domain walls are Bloch-like walls, but this information is not experimentally confirmed because the STXM spatial resolution is about $25 \mathrm{~nm}$. Rough calculations based on successive experimental images in Figs. 2(c)-2(e), as well as simulated results, lead to a domain-wall propagation speed on the order of $100 \mathrm{~m} / \mathrm{s}$. While fast domain-wall speeds have been observed in $\mathrm{Co} / \mathrm{Pt} / \mathrm{AlOx}$ systems, the results presented here are achieved with 5 orders of magnitude less current density. ${ }^{9,19}$ Note that simulations show a more dissymmetric nucleation and domain-wall propagation (Fig. 3) than in the experimental images (Fig. 2). However, such behavior may be artificially wiped out by the pump-probe nature of the measurements. The position of the magnetization nucleation inside the free layer must result from the addition of the dipolar field originating from the reference layer on the free layer and the free-layer internal demagnetization field. Indeed, starting from a parallel (P) configuration of the layer magnetizations, both these dipolar and demagnetization fields favor a magnetization reversal at the ellipse center. On the contrary, here, we can note that, starting from the antiparallel (AP) state, the nucleation may occur at the edge of the ellipse as favored by the dipolar field in this configuration. Then, the relative amplitude of the dipolar and demagnetization fields has to be quantified.

Finally, information on the free-layer magnetic behavior during the incubation time can be extracted from comparison between STXM data and simulation. Simulations in Fig. 4(c) show the out-of-plane $\left(M_{z}\right)$ and in-plane $\left(M_{x}, M_{y}\right)$ components of the free-layer magnetization during the incubation time. The prenucleation dynamics features are a small amplitude oscillatory behavior of $M_{z}$ correlated to dephased $M_{x}$ and $M_{y}$ component oscillations. $M_{x}$ and $M_{y}$ oscillation amplitudes increase continuously until domain nucleation is reached. Such evolution is typical of a current-induced magnetization precession that leads to switching over a certain magnetization tilt angle. The precessional modes may be uniform ${ }^{5}$ or nonuniform. ${ }^{16}$ Figure 4(a) corresponds to a STXM image taken at $-250 \mathrm{ps}$ where the minus sign indicates that the image was taken prior to the nucleation. The component of magnetization along the PMA axis is undoubtedly nonuniform over the pillar
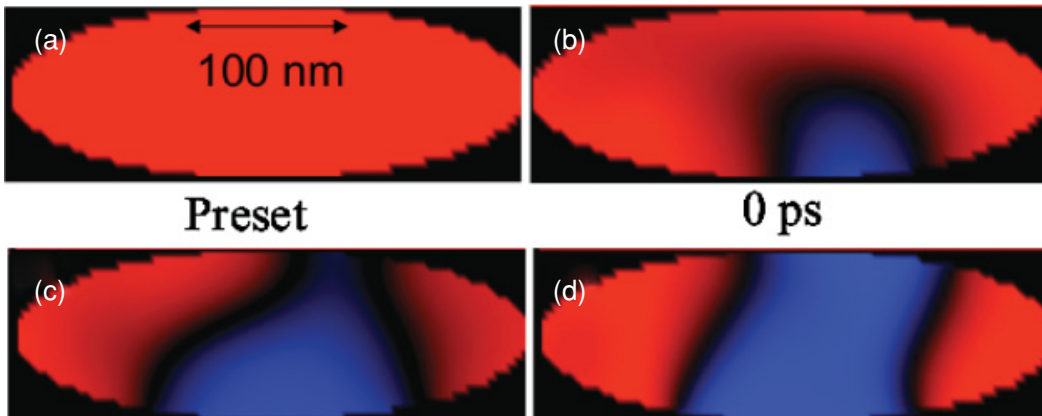

$150 \mathrm{ps}$

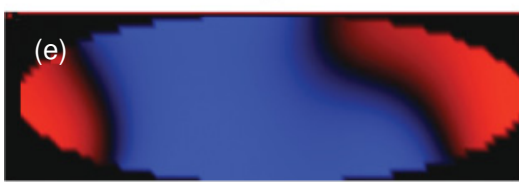

$550 \mathrm{ps}$

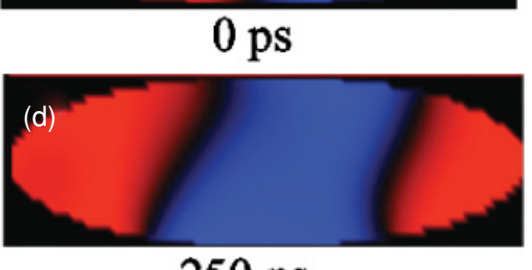

$250 \mathrm{ps}$

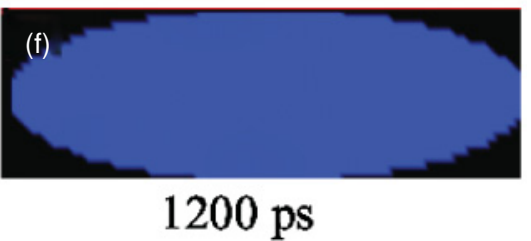

FIG. 3. (Color) A series of micromagnetic simulations showing the free-layer configuration at different times (a)-(f) during the CIMS process in a $100 \times 300 \mathrm{~nm}^{2}$ PMA nanopillar. The color scale is the same as in Fig. 2 and corresponds to the perpendicular component of the free-layer magnetization, from parallel $(\mathrm{P})(\mathrm{red})$ to AP (blue) with respect to the reference layer. Times are given with respect to domain nucleation. 

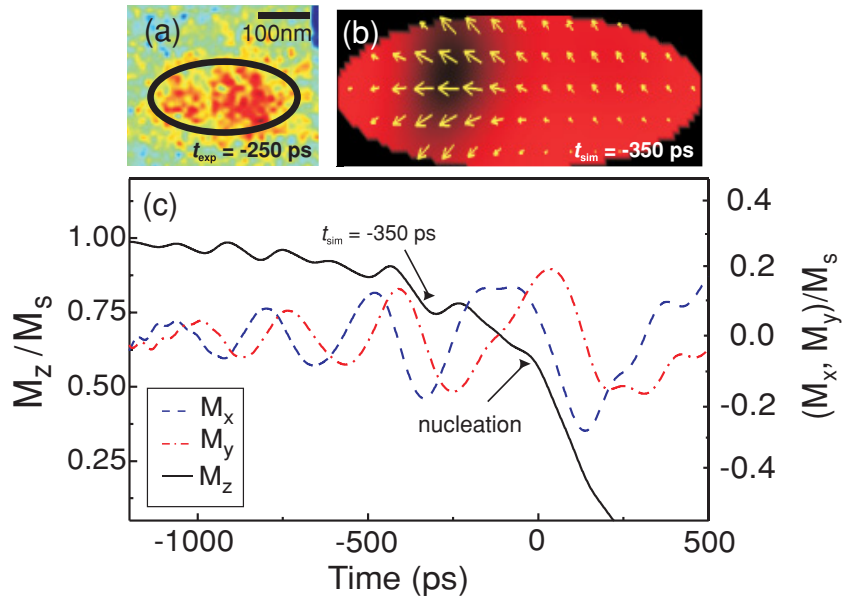

FIG. 4. (Color) (a) Experimental image of the magnetization along the PMA axis in the free layer at -250 ps where the color scale ranges from parallel (red) to AP (blue) and the negative sign indicates that the image was taken prior to nucleation. (b) The simulated magnetization in the free layer at $-350 \mathrm{ps}$ where the length of the yellow arrows corresponds to the amplitude of the in-plane magnetic component. (c) Simulated component of the magnetization along the PMA direction $\left(M_{z}\right.$, left vertical axis) as well as the two in-plane components ( $M_{x}$ and $M_{y}$, right vertical axis).

area when compared with the preset image [Fig. 2(a)]. This experimental result is well described within the simulations. A symptomatic case of the nonuniformity during the incubation time dynamics is shown in Fig. 4(b). Although all the simulated spins precess as the current is turned on, a localized area of larger amplitude precession is observed that is continuously displaced during the incubation time and ultimately results in nucleation within that region.

In conclusion, by using the nanometer-scale resolution of STXM measurements combined with time resolution given by the ALS ring, we were able to investigate CIMS dynamics driven by polarized current pulses in a PMA sample. We observed that the fast switching is highly nonuniform for a $100 \times 300 \mathrm{~nm}^{2}$ ellipse. The STXM observations are well supported using a micromagnetic calculation. Comparison with simulations provides the details of the magnetization dynamics and the nucleation/propagation process, which occur during the magnetization switching. The nonuniformities observed in the magnetization during the incubation time and as the switching occurs explain the previously reported discrepancies between experimental results and the macrospin model. ${ }^{6,7}$ Future experiments designed to study the impact of nonuniform magnetization on the switching speed and thermal stability are needed as these are critical features in the development of spin-transfer magnetic random access memory applications.

The authors would like to thank Olav Hellwig for his help with the PMA deposition process, H. Tomita, Y. Suzuki, A. Kent, and E. E. Fullerton for discussions, and the US Department of Energy, Office of Science, Office of Basic Energy Science for its continued support under Contract No. DE-AC02-76SF00515. B.B. acknowledges support from the German Research Foundation (DFG). The ALS is supported by the Director, Office of Science, Office of Basic Energy Sciences, of the US Department of Energy under Contract No. DE-AC02-05CH11231.
${ }^{1}$ D. C. Ralph and M. D. Stiles, J. Magn. Magn. Mater. 320, 1190 (2008).

${ }^{2}$ J. Katine and E. E. Fullerton, J. Magn. Magn. Mater. 320, 1217 (2008).

${ }^{3}$ S. Mangin, D. Ravelosona, J. A. Katine, M. J. Carey, B. D. Terris, and E. E. Fullerton, Nature Mater. 5, 210.

${ }^{4}$ S. Mangin, Y. Henry, D. Ravelosona, J. A. Katine, and E. E. Fullerton, Appl. Phys. Lett. 94, 012502 (2009).

${ }^{5}$ J. Z. Sun, Phys. Rev. B 62, 570 (2000).

${ }^{6}$ D. Bedau, H. Liu, J.-J. Bouzaglou, A. D. Kent, J. Z. Sun, J. A. Katine, E. E. Fullerton, and S. Mangin, Appl. Phys. Lett. 96, 022514 (2010).

${ }^{7}$ D. Bedau, H. Liu, J. Z. Sun, J. A. Katine, E. E. Fullerton, S. Mangin, and A. D. Kent, Appl. Phys. Lett. 97, 262502 (2010).

${ }^{8}$ J. Cucchiara, Y. Henry, D. Ravelosona, D. Lacour, E. E. Fullerton, J. A. Katine, and S. Mangin, Appl. Phys. Lett. 94, 102503 (2009).

${ }^{9}$ T. A. Moore, I. M. Miron, G. Gaudin, G. Serret, S. Auffret, B. Rodmacq, A. Schuhl, S. Pizzini, J. Vogel, and M. Bonfim, Appl. Phys. Lett. 93, 262504 (2008).

${ }^{10}$ Y. Acremann, V. Chembrolu, J. P. Strachan, T. Tyliszczak, and J. Stohr, Rev. Sci. Instrum. 78, 014702 (2007).
${ }^{11}$ J. P. Strachan, V. Chembrolu, X. W. Yu, T. Tyliszczak, and Y. Acremann, Rev. Sci. Instrum. 78, 054703 (2007).

${ }^{12}$ Y. Acremann, J. P. Strachan, V. Chembrolu, S. D. Andrews, T. Tyliszczak, J. A. Katine, M. J. Carey, B. M. Clemens, H. C. Siegmann, and J. Stöhr, Phys. Rev. Lett. 96, 217202 (2006).

${ }^{13}$ J. P. Strachan, V. Chembrolu, Y. Acremann, X. W. Yu, A. A. Tulapurkar, T. Tyliszczak, J. A. Katine, M. J. Carey, M. R. Scheinfein, H. C. Siegmann, and J. Stöhr, Phys. Rev. Lett. 100, 247201 (2008).

${ }^{14}$ G. Schütz, W. Wagner, W. Wilhelm, P. Kienle, R. Zeller, R. Frahm, and G. Materlik, Phys. Rev. Lett. 58, 737 (1987).

${ }^{15}$ T. Devolder, J. Hayakawa, K. Ito, H. Takahashi, S. Ikeda, P. Crozat, N. Zerounian, J.-V. Kim, C. Chappert, and H. Ohno, Phys. Rev. Lett. 100, 057206 (2008).

${ }^{16}$ Y.-T. Cui, G. Finocchio, C. Wang, J. A. Katine, R. A. Buhrman, and D. C. Ralph, Phys. Rev. Lett. 104, 097201 (2010).

${ }^{17}$ LLG micromagnetic simulator developed by Prof. M. Scheinfein, [http://llgmicro.home.mindspring.com].

${ }^{18}$ J. C. Slonczewski, J. Magn. Magn. Mater. 159, L1 (1996).

${ }^{19}$ T. Koyama, G. Yamada, H. Tanigawa, S. Kasai, N. Ohshima, S. Fukami, N. Ishiwata, Y. Nakatani, and T. Ono, Appl. Phys. Express 1, 101303 (2008). 